\title{
Ausencia segmentaria del músculo intestinal con membrana ileal en un recién nacido de extremadamente bajo peso al nacer. A propósito de un caso Segmental absence of intestinal muscle with ileal web in an extremely low birth weight infant. Case report
}

\author{
Dr. Mehmet Buyuktiryaki ${ }^{a}$, Dra. Hayriye G. Kanmaz ${ }^{a}$ Dra. Nilüfer Okur ${ }^{a}$, Dr. Ufuk Ates ${ }^{b}$, \\ Dr. Ali L. Sirvan ${ }^{c}$ y Dra. Nurdan Uras ${ }^{a}$
}

\begin{abstract}
RESUMEN
Las perforaciones intestinales espontáneas son perforaciones localizadas sin las características clínicas, radiológicas e histopatológicas típicas de la enterocolitis necrosante. La perforación intestinal espontánea es una entidad clínica de reciente definición. El factor de riesgo más conocido es la prematurez. Se presenta en el 2-3\% de los recién nacidos de muy bajo peso al nacer y en el $5 \%$ de los neonatos de extremadamente bajo peso. En este artículo presentamos el caso de un recién nacido de extremadamente bajo peso al nacer con perforación intestinal espontánea, ausencia segmentaria del músculo intestinal y membrana ileal como causas subyacentes. Nuestro objetivo es llamar la atención a la ausencia segmentaria del músculo intestinal, una afección rara pero que se informa cada vez más como causa de perforación intestinal espontánea, y a la importancia del examen histopatológico de las muestras obtenidas durante una cirugía.

Palabras clave: perforación intestinal espontánea, recién nacido prematuro, músculo intestinal, membrana ileal.
\end{abstract}

http:/ /dx.doi.org/10.5546/aap.2016.e108

\section{INTRODUCCIÓN}

La perforación intestinal espontánea (PIE) es una perforación intestinal única que suele afectar el borde antimesentérico del íleon distal y que cursa sin pródromo definido en recién nacidos extremadamente prematuros durante las primeras dos semanas de vida. Su incidencia es del 2-3\% en los recién nacidos de muy bajo peso al nacer (MBPN) y del $5 \%$ en los neonatos de

a. División de Neonatología, Hospital Universitario Maternal Zekai Tahir Burak, Ankara, Turquía.

b. División de Cirugía Pediátrica, Hospital Universitario Maternal Zekai Tahir Burak, Ankara, Turquía.

c. División de Patología, Hospital Universitario Maternal Zekai Tahir Burak, Ankara, Turquía.

Correspondencia:

Dr. Mehmet Buyuktiryaki: mbuyuktiryaki@yahoo.com

Financiamiento: ninguno.

Conflicto de intereses: ninguno que declarar.

Recibido: 3-8-2015

Aceptado: 15-10-2015 extremadamente bajo peso al nacer (EBPN). ${ }^{1} \mathrm{El}$ factor de riesgo más conocido es la prematurez. ${ }^{2}$ La presencia de una membrana intestinal es una anomalía congénita poco frecuente y que suele informarse durante la niñez. ${ }^{3}$ De manera similar, la ausencia segmentaria del músculo intestinal (ASMI) es también una afección rara y aún no se ha elucidado completamente su patogenia. En general, suelen presentarse perforación intestinal o síntomas de obstrucción. La ausencia de músculo en cualquier porción del aparato intestinal produce obstrucción debido a la ausencia de movimientos peristálticos y la dilatación del segmento afectado. En cuanto a la patogenia, no es claro si la perforación se debe principalmente a la malformación o es secundaria a la isquemia y la inflamación. La isquemia y la hipoxia son las principales etiologías posibles, dado que la mayoría de los casos se presentan en recién nacidos con síndrome de dificultad respiratoria (SDR) y muy bajo peso al nacer.

En este artículo presentamos el caso de un recién nacido de EBPN con SDR sometido a cirugía por perforación intestinal espontánea debido a ausencia segmentaria del músculo intestinal acompañada por una membrana ileal.

\section{Caso clínico}

Un varón nació con $815 \mathrm{~g}$, a las $24^{5 / 7}$ semanas de gestación por parto vaginal espontáneo tras el primer embarazo de una madre de 23 años de edad. La madre no había recibido los controles prenatales adecuados; ingresó a la sala de emergencias con contracciones y trabajo de parto prematuro. En la ecografía obstétrica de emergencia se observó polihidramnios; por lo demás, el neonato estaba sano. La madre dio a luz poco tiempo después de ingresar. Se realizó la reanimación neonatal y se intubó al recién nacido en la sala de partos. Su puntaje de Apgar fue 3 al primer minuto y 7 al quinto minuto. $\mathrm{Al}$ momento del ingreso a la unidad de cuidados 
intensivos neonatales (UCIN), los signos vitales eran frecuencia cardíaca de 145/minuto, presión arterial de 57/39 (45) mmHg y saturación del $94 \%$. Al paciente se le administró surfactante (100 mg/kg de beractant) debido a los hallazgos clínicos y radiológicos de síndrome de dificultad respiratoria. Se le colocaron catéteres en arteria y vena umbilicales. Se le administró una dosis de carga de cafeína $(20 \mathrm{mg} / \mathrm{kg}$ ) por vía intravenosa; la dosis de mantenimiento fue de $5 \mathrm{mg} / \mathrm{kg} /$ día. Expulsó el meconio a las 42 horas de vida y presentaba ruidos hidroaéreos audibles; se inició alimentación enteral (15 cc/ kg/día) junto con leche materna. A las 70 horas de vida presentó residuos que contenían bilis. En la radiografía abdominal simple se observó dilatación de las asas intestinales, aunque no se observaron aire libre, niveles hidroaéreos ni neumatosis intestinal. Se le retiró la alimentación enteral y se realizó una descompresión orogástrica. Excepto por la intolerancia a la alimentación, el paciente se encontraba bien. A los 5 días de vida, presentó una repentina distensión abdominal significativa, con coloración azulada de la piel del abdomen. En la radiografía abdominal simple de pie se observó aire libre debajo del diafragma. Debido a que no era posible descartar una septicemia intrahospitalaria, se le administró un tratamiento antibiótico empírico.

La condición general del paciente era mala, presentaba hiperglucemia e insuficiencia renal aguda y estaba hemodinámicamente inestable.

FIgURA 1. Perforación del íleon

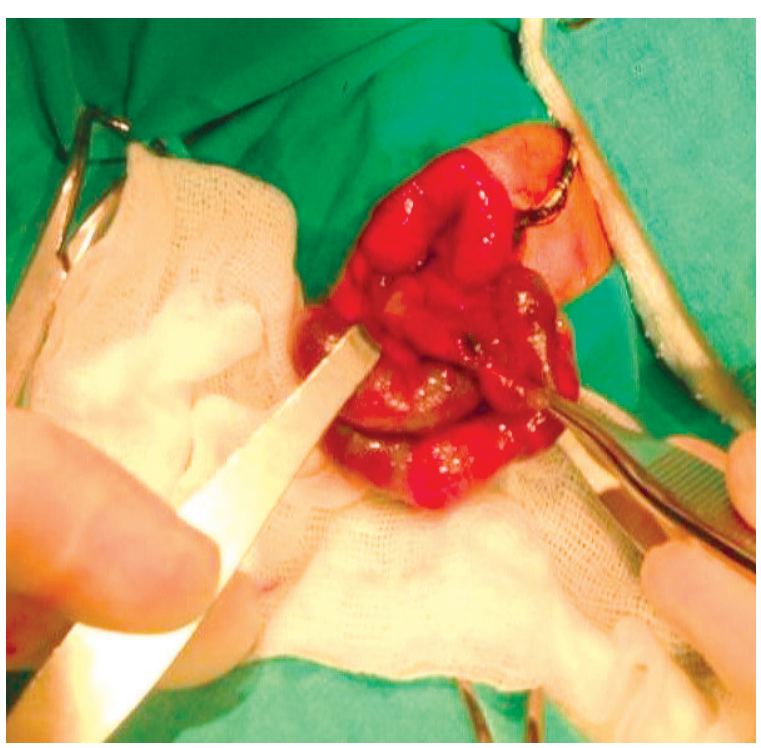

Se le realizó un drenaje peritoneal primario (DPP). Al octavo día de vida, el estado clínico había mejorado, pero continuaba con la sonda orogástrica y el drenaje. Cuando el paciente comenzó a defecar, se le retiró el drenaje; defecaba en forma espontánea y estaba hemodinámicamente estable. No obstante, a los 11 días de vida, presentó distensión abdominal y se le detectó aire libre debajo del diafragma mediante radiografía abdominal; por lo tanto, se lo sometió a una cirugía. Se halló un segmento de $20 \mathrm{~cm}$ del íleon colapsado, próximo al ciego. También se localizaron dos perforaciones del íleon a 30 y 40 centímetros, próximas al ciego. Se resecó el segmento perforado y se realizó la anastomosis de ambos extremos. En el examen macroscópico, el intestino era normal, excepto por las dos perforaciones antes mencionadas (Figura 1). Se realizó una incisión en la unión del íleon normal y del colapsado y se colocó una sonda de alimentación de 8 Fr a través del íleon colapsado; se halló una membrana intestinal a $5 \mathrm{~cm}$ en sentido distal. Se resecó un segmento de $6 \mathrm{~cm}$ del íleon, junto con la membrana. Después de la resección, se unieron los extremos del íleon remanente mediante anastomosis. En el examen histopatológico de los segmentos ileales resecados se observaron mucosa intestinal normal y submucosa edematosa, con leve infiltración de linfocitos junto con ausencia segmentaria del músculo intestinal (Figura 2). La membrana serosa era normal y no presentaba necrosis. El paciente falleció a los 15 días de vida
FIgURA 2. Mucosa intacta y submucosa edematosa con infiltración leve de linfocitos junto con ausencia segmentaria de la capa muscular (HEE 20X)

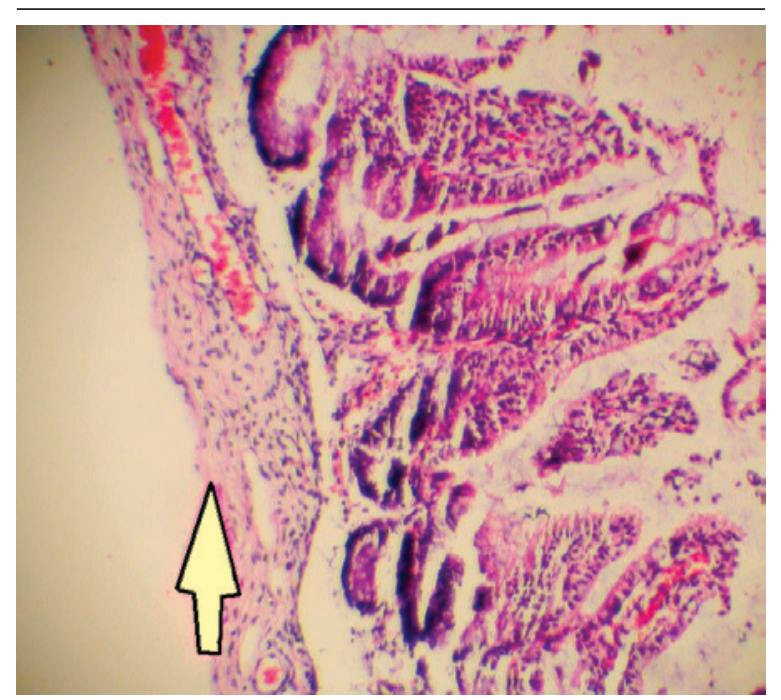


debido a septicemia e insuficiencia multiorgánica. Los padres no otorgaron el consentimiento para una autopsia, por lo que no se realizó.

\section{DISCUSIÓN}

Aún se desconoce la etiología de la PIE. Si bien se ha notificado previamente el adelgazamiento o la ausencia de la capa muscular en el lugar de la perforación, de manera similar a nuestro caso, no es claro si estos cambios participan en la patogenia de la PIE. Además, en nuestro paciente se halló una membrana ileal. Ambas patologías podrían estar causadas por compresión vascular in utero que produce una menor perfusión intestinal e isquemia del segmento intestinal correspondiente, lo que deriva en que los intestinos sean susceptibles a la perforación. ${ }^{4,5}$

En la bibliografía se han informado varios casos de PIE con anomalías del músculo intestinal observadas en el examen histopatológico. ${ }^{6}$ Hasta donde sabemos, este es el primer caso en la bibliografía donde se presenta ASMI y membrana ileal como causas subyacentes de la PIE. Muchos autores han destacado que la ausencia de músculo no es una malformación congénita, sino secundaria al daño isquémico; sin embargo, aún no es claro por qué la mucosa, que es más vulnerable a la isquemia que el músculo, permanece intacta. ${ }^{7}$ Se conoce que la capacidad de regeneración de la mucosa y de los músculos es diferente, por lo que la capa mucosa se regenera tras una isquemia, pero la capa muscular no logra regenerarse y se produce una ausencia aislada de músculo. Es posible especular que la membrana en nuestro paciente se formó como resultado de la exagerada respuesta regenerativa a la isquemia. ${ }^{6}$

La ASMI se presenta sin síntomas de obstrucción. El polihidramnios, que se informó en este paciente, podría indicar obstrucción intestinal in utero, a pesar de que en la ecografía prenatal no se observaron asas dilatadas ni aumento de la ecogenicidad. La presencia de ruidos hidroaéreos normales y la distribución de gas hacia el recto observadas en la radiografía simple, y la defecación espontánea en los tres primeros días de vida indicaban una obstrucción parcial y fueron la causa de la manifestación atípica. La ausencia segmentaria del músculo intestinal es una afección clínica rara; sin embargo, su presentación clínica y avance son similares a la presentación frecuente de la peritonitis. La laparotomía exploratoria es el tratamiento de referencia en estos escenarios clínicos, y el diagnóstico depende de la histopatología de las muestras resecadas. ${ }^{8}$ El drenaje peritoneal primario se usa cada vez más frecuentemente como tratamiento inicial de la PIE en las UCIN dado que puede realizarse junto a la cama del paciente y los resultados son similares a la laparotomía exploratoria..$^{9,10}$ No obstante, no permite obtener muestras intestinales y las causas subyacentes, como la ASMI, podrían no establecerse mediante esta técnica.

La ausencia segmentaria del músculo intestinal representa una entidad diferente, que podría presentarse en diversas formas. Este es el primer informe que describe su asociación con una membrana ileal y podría respaldar la teoría que propone una isquemia intrauterina focal durante el desarrollo del aparato gastrointestinal como etiología. Es necesario considerar la biopsia y la evaluación patológica al momento de la cirugía.

\section{REFERENCIAS}

1. Meyer CL, Payne NR, Roback SA. Spontaneous, isolated intestinal perforations in neonates with birth weight less than 1,000 g not associated with necrotizing enterocolitis. J Pediatr Surg 1991;26(6):714-7.

2. Pumberger W, Mayr M, Kohlhauser C, Weninger M. Spontaneous localized intestinal perforation in very-lowbirth-weight infants: a distinct clinical entity different from necrotizing enterocolitis. J Am Coll Surg 2002;195(6):796-803.

3. Best KE, Tennant PW, Addor MC, Bianchi F, et al. Epidemiology of small intestinal atresia in Europe: a register-based study. Arch Dis Child Fetal Neonatal Ed 2012;97(5):F353-8.

4. Tatekawa Y, Muraji T, Imai Y, Nishijima E, et al. The mechanism of focal intestinal perforations in neonates with low birth weight. Pediatr Surg Int 1999;15(8):549-52.

5. JainS, Wadhwa N, MunjalS. Segmental absence of intestinal musculature with intestinal atresia and ileal perforation. Pathology 2009;41(6):596-8.

6. Oretti C, Bussani R, Janes A, Demarini S. Multiple segmental absence of intestinal musculature presenting as spontaneous isolated perforation in an extremely lowbirth-weight infant. J Pediatr Surg 2010;45(8):E25-7.

7. Stephens D, Arensman R, Pillai S, Alagiozian-Angelova $\mathrm{V}$. Congenital absence of intestinal smooth muscle: a case report and review of the literature. I Pediatr Surg 2009;44(11):2211-5.

8. Eicher C, Seitz G, Bevot A, Moll M, et al. Surgical management of extremely low birth weight infants with neonatal bowel perforation: a single-center experience and a review of the literature. Neonatology 2012;101(4):285-92.

9. Moss RL, Dimmitt RA, Barnhart DC, Sylvester KG, et al. Laparotomy versus peritoneal drainage for necrotizing enterocolitis and perforation. $N$ Engl J Med 2006;354(21):2225-34.

10. Blakely ML, Lally KP, McDonald S, Brown RL, et al. Postoperative outcomes of extremely low birth-weight infants with necrotizing enterocolitis or isolated intestinal perforation: a prospective cohort study by the NICHD Neonatal Research Network. Ann Surg 2005;241(6):984-9. 